\title{
The effects of respiratory infections, atopy, and breastfeeding on childhood asthma
}

\author{
W.H. Oddy*,\#, N.H. de Klerk*, P.D. Sly*, , P.G. Holt*
}

The effects of respiratory infections, atopy, and breastfeeding on childhood asthma. W.H. Oddy, N.H. de Klerk, P.D. Sly, P.G. Holt. (C) ERS Journals Ltd 2002.

ABSTRACT: The objectives of the present study were to quantify the association of atopy and respiratory infections with asthma, and exclusive breastfeeding with respiratory illness, atopy and asthma in children.

A cohort study of 2,602 children enrolled prior to birth and followed prospectively, provided data on respiratory illness, the method of feeding in the first year of life, as reported on a prospective diary card, and current asthma at the age of 6 yrs (defined as doctor-diagnosed asthma with wheeze in the last year or cough without a cold, and currently taking either preventer or reliever asthma medication), as reported by parental questionnaire. Atopy was defined by a positive skin-prick test assessed at the age of 6 yrs.

Wheezing lower respiratory illness (LRI) in the first year of life, particularly multiple episodes of wheezing LRI, increased the risk for current asthma in both nonatopic (odds ratio (OR) 4.10, $\mathrm{p} \leqslant \mathbf{0 . 0 0 0 5}$ ) and atopic children (OR 9.00, $\mathrm{p} \leqslant \mathbf{0 . 0 0 0 5}$ ), but did not increase the risk for atopy. In contrast, up to three upper respiratory tract infections demonstrated a negative association and four or more a positive risk for current asthma in unadjusted $(\mathrm{p}=\mathbf{0 . 0 0 6})$ and adjusted $(\mathrm{p}=\mathbf{0 . 0 5 7})$ analysis. Following adjustment, exclusive breastfeeding for $<4$ months was associated with an increased risk for current asthma (OR 1.36, 95\% confidence interval 1.00-1.85, $\mathrm{p}=0.047$ ).

Wheezing lower respiratory illness in the first year of life and atopy are independently associated with increased risk for current asthma at the age of 6 yrs, suggesting that their effects are mediated via different causal pathways and that these risk factors are multiplicative when they operate concomitantly within individual children. Exclusive breastfeeding protects against asthma via effects on both these pathways, as well as through other as yet undefined mechanisms.

Eur Respir J 2002; 19: 899-905.
*Telethon Institute for Child Health Research, Centre for Child Health Research, Faculty of Medicine and Dentistry, University of Western Australia, " Dept of Nutrition, Dietetics and Food Science, School of Public Health, Curtin University of Technology and Princess Margaret Hospital for Children, Perth, Australia.

Correspondence: W.H. Oddy

Telethon Institute for Child Health Research

PO Box 855 West Perth

Western Australia 6872

Australia

Fax: 61894897700

E-mail: wendyo@ichr.uwa.edu.au

\section{Keywords: Atopy}

breastfeeding

childhood asthma

respiratory infections

Received: December 112000

Accepted after revision January 17 2002
The aetiology of childhood asthma is not fully understood but reported risk factors include male sex, low birth weight, preterm birth, young maternal age, maternal asthma and parental smoking [1-6]. Exposure to high levels of inhalant allergens, including house dust mites [7] or pollens, during infancy are risk factors for atopic sensitization, which is also strongly associated with asthma risk. Early exposure to certain respiratory infections may be protective for atopy and/or asthma, whereas some infections have been suggested to exert the opposite effects $[8,9]$. Furthermore, exclusive breastfeeding may protect against asthma and reduce the incidence of lower respiratory illness (LRI), especially respiratory syncytial virus (RSV) [10-14]. The extent to which these factors interact is unclear.

Breastfeeding has been shown to protect against infection in infants $[15,16]$, but protection against respiratory illnesses has not been consistently demonstrated $[17,18]$. Differences in study design, analytical methods and possible biases have complicated the interpretation of many studies.

A protective effect of exclusive breastfeeding on asthmatic traits at the age of 6 yrs [10] has previously been demonstrated. The aim of this study was to clarify, in the same cohort, whether this protective association resulted from intermediate associations with respiratory infections, atopy or other facets of breastfeeding. The study's objectives were to quantify the association of atopy and respiratory infections with asthma, and exclusive breastfeeding with respiratory illness, atopy and asthma in children.

\section{Methods}

\section{Study population}

The Western Australian Pregnancy Cohort Study was established between 1989-1992 to investigate several hypotheses about antenatal and perinatal antecedents of disease. The cohort was recruited serially from the public antenatal clinic at the King Edward Memorial Hospital or a nearby private practice in Perth [19]. A total of 2,979 Western Australian children were enrolled by 18 weeks 
gestation. At the time of enrolment, parents of the study children completed a questionnaire about their own respiratory illness, smoking behaviour and general health.

By the end of the pregnancy phase, 2,888 mothers remained in the study. Of the 2,860 live births, 13 children had since died, 124 children had been withdrawn, and 121 children (predominantly living overseas) were lost to follow-up. Thus, 2,602 of 2,860 $(91 \%)$ children remained available for follow-up at the age of 6 yrs.

The mothers received antenatal care and gave birth at the King Edward Memorial Hospital. The babies were examined at the age of 2 days by a paediatrician or trained midwife who was unaware of the information collected during the antenatal period. Informed consent was obtained for follow-up of the children. A detailed record of perinatal morbidity was obtained for each baby, and mothers were issued with a diary at discharge in which to record episodes of illness, feeding history and other key events in the first year of the infant's life.

\section{Assessment at 1 yr}

Parents kept a diary of their child's illnesses and feeding history and completed a standardized questionnaire at the end of the first year, in which they were asked to record details about any illnesses or problems that required a visit to the clinic or doctor and infant feeding (age of children at completion of the 1-yr questionnaire (mean \pm SD) $13.9 \pm 1.4$ months). Parents subsequently brought their child to the hospital for assessment by a paediatrician and research nurse. At the assessment, the research nurse verified feeding history and recorded illnesses from the diary card, and then transcribed the details onto the questionnaire using International Classification of Disease (ICD)-9 codes to classify illness. At the end of the first year, 2,411 questionnaires were completed ( $84 \%$ of the initial cohort, $92 \%$ of those consenting to follow-up) and 2,365 (82\%, 90\%) attended for clinical assessment.

\section{Assessment at 6 yrs}

Shortly before their child's sixth birthday (mean age $71 \pm 6.6$ months), the parents of cohort children were contacted and a further questionnaire was mailed. Among other questions, parents were asked about the history of respiratory symptoms and illness in the study child. Complete questionnaire data were available from 2,173 children representing a $76 \%$ response rate. Clinical assessment data provided skin-prick test results from 1,595 children $(56 \%)$ or $79 \%$ of the 2,012 children clinically examined at the age of 6 yrs.

\section{Outcome}

The expression of asthma symptoms changes during the preschool years and the diagnosis of asthma is a clinical one. Consequently, a stringent phenotypic definition was applied for current asthma at the age of 6 yrs (defined as doctor-diagnosed asthma-ever with wheeze or cough without a cold in the last year, and currently taking either preventer or reliever asthma medication).

\section{Exposures}

Respiratory illnesses (both upper and lower) were considered as exposure variables for current asthma. The number of upper respiratory tract infections (including tonsillitis, otitis media or otitis media with effusion), wheezing LRI (including bronchiolitis, bronchospasm or asthma) and nonwheezing LRI (including chest infection, pneumonia, whooping cough, chronic cough or croup) in the first year of life were explored as risk factors for current asthma.

Atopy was also considered as an exposure variable for current asthma. Skin-prick testing to four common aeroallergens (standardized mite, standardized cat pelt, ryegrass (perennial lolium perenne) and mould mix \#4 (Bayer Corporation, Pharmaceutical Division, Elkhart, IN, USA)) was conducted as described by DREBORG [20] when the children attended the 6-yr clinical assessment. Atopy was defined by a positive skin-prick test if the weal to at least one of the four common environmental aeroallergens was $\geqslant 2 \mathrm{~mm}$ and larger than that of a control, $10 \mathrm{~min}$ after the test. A weal size of $2 \mathrm{~mm}$ rather than $3 \mathrm{~mm}$ was chosen both because of the young age of the children and in order to incorporate all cases, including those with milder atopy. Analyses that included atopy were restricted to those children with skin tests.

Early infant feeding was measured as the duration of exclusive breastfeeding in the first year and was recorded as an eight-level categorical variable (table 1), but for consistency was included as a dichotomous variable (exclusively breastfed $<4$ months, exclusively breastfed $\geqslant 4$ months) in most of the analyses. The authors have previously demonstrated a protective effect of exclusive breastfeeding on asthmatic traits in $6 \mathrm{yr}$ olds in a dose/response effect that was robust to the choice of cut-off point at the ages of 3, 4, 5 and 6 months, exclusive breastfeeding being defined as no introduction of "other milk" [10].

The potential confounding exposures were sex, gestational age, maternal smoking in pregnancy and maternal history of asthma. Other factors considered in the analysis included maternal age, education, the age that solids were introduced, family income and infection exposure (early childcare and older siblings). Only variables with a consistently significant association to the outcome were included in the multivariate analyses.

\section{Statistical analysis}

Unconditional logistic regression was used to assess multiple effects. The study had $>95 \%$ power to detect an odds ratio (OR) of 1.5 for most analyses. 
Table 1.-Observed prevalence of asthma and atopy outcomes and selected exposure characteristics

\begin{tabular}{lc}
\hline Outcomes and exposure characteristics & Prevalence \% \\
\hline Subjects n & 2456 \\
Primary outcome at 6 yrs of age & \\
Current asthma & 18.0 \\
Primary exposures & \\
Positive skin-prick test at 6 yrs & 41.4 \\
Upper respiratory tract & \\
infections in first year & \\
0 & 40.5 \\
1 & 24.4 \\
$2-3$ & 22.7 \\
$\geqslant 4$ & 12.4 \\
Wheezing lower respiratory & \\
tract illness in first year & 74.9 \\
0 & 13.3 \\
1 & 11.8 \\
$\geqslant 2$ & \\
Nonwheezing lower respiratory & \\
tract illness in first year & \\
0 & 10.7 \\
1 & 3.5 \\
$\geqslant 2$ &
\end{tabular}

Other exposures

Duration of exclusive

breastfeeding months

Never breastfed

1

2

3

4

5

$\geqslant 7$

Male sex

Gestational age of $<37$ weeks

Maternal smoking at any

stage of pregnancy

Older siblings

Childcare before the age of 3 months

Maternal asthma (current)

\#: Data for 387 of 2,155 subjects; ${ }^{\top}$ : a weal size $\geqslant 2 \mathrm{~mm}$ (data for 660 of 1,595 subjects).

\section{Ethics approval}

Informed consent was obtained for follow-up of the children from birth. The Ethics Committees of the King Edward and Princess Margaret Hospitals approved the protocol for the study.

\section{Results}

Childhood asthma, atopy and respiratory illness during infancy

Current asthma and atopy were reported in 18 and $41 \%$ of children, respectively (table 1 ), and were strongly related (table 2 ).

A small protective association of one to three upper respiratory tract infections with current asthma was evident, whereas four or more constituted a risk. However, these associations were of marginal
Table 2. - Current asthma and atopy

\begin{tabular}{lcc}
\hline & \multicolumn{2}{c}{ Current asthma } \\
\cline { 2 - 3 } & No & Yes \\
\hline Atopic status & & \\
no & $769(85.9)^{\#}$ & $126(14.1)^{\#}$ \\
yes & $478(75.3)^{\bullet}$ & $157(24.7)^{\bullet}$ \\
\hline
\end{tabular}

Data are presented as $\mathrm{n}(\%)$. Odds ratio $2.01 ; 95 \%$ confidence interval $1.55-2.60$; p-value $\leqslant 0.0005$. \#: $n=895$; : $\mathrm{n}=635$.

significance after adjustment (table 3 ). The relationship between wheezing LRI and current asthma was clearer, with an increased risk of current asthma observed with one or more wheezing LRI in the first year of life $(p<0.0005)$. One nonwheezing LRI or infection was significantly associated with current asthma in unadjusted and adjusted analyses, whereas two or more were not.

Neither upper (one or more), wheezing (one or more) or nonwheezing (one) LRI appeared to significantly influence the risk of atopy. However, two or more nonwheezing LRI demonstrated a protective relationship for atopy that did not reach statistical significance (OR $0.61,95 \%$ confidence interval (CI) $0.34-1.10, p=0.102)$.

Separate analyses were performed with atopy status at the age of 6 yrs and were considered to be exposure variables for current asthma in a multivariate model with wheezing LRI and the other identified confounders. There was no evidence of statistical interactions between atopy and wheezing LRI

Table 3. - Association between current asthma in children aged 6 yrs and respiratory infection in the first year of life

\begin{tabular}{|c|c|c|}
\hline & \multicolumn{2}{|c|}{ Current asthma ${ }^{\#}$} \\
\hline & Unadjusted & Adjusted \\
\hline \multicolumn{3}{|c|}{ Upper respiratory } \\
\hline \multicolumn{3}{|c|}{ tract infections } \\
\hline 0 & 1.00 & 1.00 \\
\hline 1 & $0.78(0.61-1.00)$ & $0.99(0.73-1.34)$ \\
\hline $2-3$ & $0.73(0.56-0.97)$ & $0.75(0.54-1.04)$ \\
\hline$\geqslant 4$ & $1.57(1.10-2.25)$ & $1.32(0.92-1.89)$ \\
\hline p-value ${ }^{+}$ & 0.006 & 0.057 \\
\hline \multirow{2}{*}{\multicolumn{3}{|c|}{$\begin{array}{l}\text { Wheezing lower } \\
\text { respiratory tract illness }\end{array}$}} \\
\hline & & \\
\hline 0 & 1.00 & 1.00 \\
\hline 1 & $1.61(1.15-2.24)$ & $1.44(1.02-2.04)$ \\
\hline$\geqslant 2$ & $3.35(2.46-4.56)$ & $3.84(2.82-5.24)$ \\
\hline p-value ${ }^{+}$ & $<0.0005$ & $<0.0005$ \\
\hline \multicolumn{3}{|c|}{$\begin{array}{l}\text { Nonwheezing lower } \\
\text { respiratory tract illness }\end{array}$} \\
\hline 0 & 1.00 & 1.00 \\
\hline 1 & $1.61(1.17-2.23)$ & $1.46(1.04-2.07)$ \\
\hline$\geqslant 2$ & $1.30(0.71-2.38)$ & $1.07(0.56-2.04)$ \\
\hline p-value ${ }^{+}$ & 0.012 & 0.093 \\
\hline
\end{tabular}

Data are presented as odds ratio (95\% confidence interval). \#. data from 387 of 2,155 subjects; ${ }^{+}$: p-value for heterogeneity of odds ratio; ": adjusted for all types of infections as well as sex, gestational age $<37$ weeks and maternal smoking at any stage of pregnancy. 
Table 4. - Association of combinations of wheezing lower respiratory illness and atopic status (as ascertained by skin-prick test positivity) with current asthma

\begin{tabular}{lcccc}
\hline & \multicolumn{2}{c}{ Current asthma } \\
\cline { 2 - 5 } & Skin-prick test negative & p-value & Skin-prick test positive & p-value \\
\hline Wheezing lower respiratory illness & & & & \\
0 & 1.00 & & $2.30(1.62-3.29)$ & 0.000 \\
1 & $2.03(1.19-3.45)$ & 0.010 & $3.39(1.91-6.01)$ & 0.0004 \\
$\geqslant 2$ & $4.10(2.48-6.77)$ & $<0.0005$ & $9.00(5.25-15.42)$ & $<0.0005$ \\
\hline
\end{tabular}

Data are presented as odds ratio ( $95 \%$ confidence interval), adjusted for duration of exclusive breastfeeding, sex, gestational age $<37$ weeks and maternal smoking during pregnancy. Data for 221 of 1,339 subjects are presented, the sample is reduced due to missing data.

( $\mathrm{p}=0.701)$, atopy and breastfeeding $(\mathrm{p}=0.982)$ or breastfeeding and wheezing LRI $(\mathrm{p}=0.588)$ in current asthma, implying that all effects were acting independently on a multiplicative scale. As illustrated in table 4, expression of the atopic phenotype, as defined by skin-prick test positivity, was associated with an increased risk of current asthma at 6 yrs of age depending on wheezing LRI status. It is clear that the highest risk group for current asthma were those children who had two or more wheezing LRI and were skin-prick test positive. In this highest-risk group, breastfeeding exclusively for $<4$ months increased the risk for current asthma further (OR 2.39, 95\% CI $1.47-3.89, \mathrm{p}<0.0005)$.

\section{Childhood asthma, infant feeding, respiratory illness and atopy}

The following were identified as a significant risk factors for wheezing LRI in the first year of life: cessation of exclusive breastfeeding before the age of 6 months (OR 1.98, 95\% CI 1.46-2.68, $\mathrm{p} \leqslant 0.0005$ ) (table 5); male sex (one wheezing LRI: OR 1.48, 95\% CI 1.16-1.90, $\mathrm{p}=0.002$; two or more wheezing LRIs: OR $1.35,95 \%$ CI $1.04-1.77, \mathrm{p}=0.025)$; and maternal smoking during pregnancy (one wheezing LRI: OR $1.12,95 \%$ CI $0.86-1.44, \mathrm{p}=0.403$; two or more wheezing LRIs: OR 1.46, 95\% CI 1.12-1.91, $\mathrm{p}=0.005$ ). The cessation of breastfeeding before the age of 2 months was a significant risk factor for four or more upper respiratory tract infections in the first year of life (OR 1.40, 95\% CI 1.04-1.88, $\mathrm{p}=0.028$ ). There was no association of infant feeding with the occurrence of nonwheezing LRI in these data.

In multivariate analyses, exclusive breastfeeding for $<4$ months showed a weak association with current asthma (OR 1.19, $\mathrm{p}=0.170)$ (table 6). Following adjustment for atopy, a shorter duration of exclusive breastfeeding continued to be a risk factor, although it was not significant (OR 1.32, $\mathrm{p}=0.067$ ). The association weakened when only infections were included in the model (OR 1.18, $\mathrm{p}=0.194)$. However, when all confounders were included, the risk of exclusive breastfeeding for $<4$ months increased (OR 1.32, $\mathrm{p}=0.066$ ) and reached statistical significance when maternal asthma status was added to the model (OR 1.36, $\mathrm{p}=0.047$ ). Although these associations were of marginal statistical significance, evidenced by their CI sizes, they were potential risk factors in each model. Further, there was no significant interaction between maternal asthma and breastfeeding in the association with current asthma $(\mathrm{p}=0.465)$.

\section{Discussion}

The Western Australian Pregnancy Cohort Study has previously been shown to be reasonably representative of the general Western Australian population [19]. Although random nonresponse may have reduced statistical power, the reported power calculations in this study are based upon the final sample size and take nonresponse into account, so the positive conclusions remain valid. Any systematic nonresponse is likely to be determined by disease status and social class, which may have biased estimated effects in either direction. However, the addition of social-class covariates to the models made little difference to the point estimates. Mothers were enrolled in the study in midpregnancy (before any foetal outcomes were known), leaving little scope for selection bias in relation to asthma or atopy. Drop-out at birth due to early death or loss to follow-up was rare $(0.4 \%)$ and the study population was large with a high response rate.

Recruitment of the Western Australian Pregnancy Cohort Study was mainly through a tertiary obstetric hospital and included a small excess of mothers with preterm babies. The present study models included a covariate reflecting preterm delivery and confounding due to "at-risk" pregnancy, which should not have distorted the conclusions. The data set provided adequate power and model checking, suggesting that the mathematical models gave acceptable representation of the observed data. One of the strengths of the study was that exposure data were recorded prospectively by the parent on a diary card, thereby reducing recall of respiratory illness that may be more pronounced in mothers with asthma. In addition, measurement of outcome data was based on validated questionnaires and methodologies, and, although based on selfreport, included the criterion that medication be used.

The effect of early infections on current asthma was related to the location, intensity and frequency of the infections. Thus, having up to three upper respiratory tract infections in the first year of life was associated 


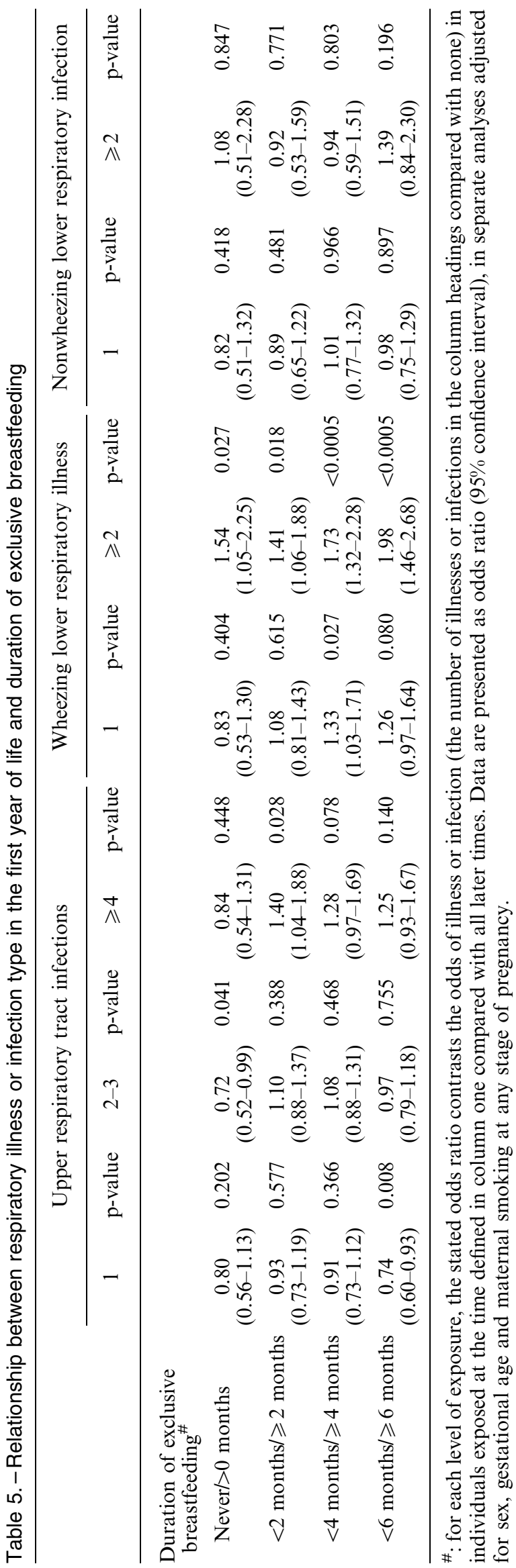

with a modest reduction in risk of current asthma at an age of 6 yrs, whereas more frequent infections appeared to increase the risk. In contrast, the occurrence of any lower respiratory tract infections was associated with current asthma at an age of 6 yrs, particularly if infections were associated with wheeze. The risk was markedly increased if they occurred more than once before the child's first birthday or if the child was atopic.

In this study, it was evident that the risk associated with wheezing LRI was greatest amongst atopics, as has been reported previously [21]. These illnesses equally enhanced the risk of subsequent asthma in nonatopic children, as has been observed in other studies $[22,23]$. Further, this protective effect was the same in the present study, irrespective of maternal history of asthma, contrary to the findings of others [5]. With regard to the potential role of atopy in asthma development, it has previously been demonstrated that during early childhood, long-term immunological memory is developed against common inhalant allergens, and the nature of the cytokines, which dominate these memory responses, determines whether subjects will manifest the atopic or nonatopic responder phenotype [24]. The levels of atopic sensitization to inhalant allergens at this age are likely to cause only moderate airway inflammation, which occasionally exceeds the threshold required to trigger wheeze. The relatively low risk of asthma associated with atopy in this cohort (OR 2.01, 95\% CI 1.55-2.60) would suggest that the level of inflammation achieved is usually insufficient to cause permanent changes in the airway tissues.

LRI can also induce airways inflammation and accompanying wheeze, and severe infections potentially cause inflammatory responses, which are considerably more intense than those triggered by exposure to allergens. Infections of this nature which occur during the phase of rapid lung growth in infancy, have been hypothesized to have severe sequelae in relation to respiratory function in the short-to-medium term, as they can potentially cause phenotypic changes within the growing airway that induce a state of hyperreactivity to extraneous irritant stimuli $[6,25]$. However, if exposure to such secondary airborne stimuli is intermittent, overt symptoms may again be restricted to occasional wheeze. The outcome, however, may be quantitatively different if the secondary airborne stimuli are inhalant allergens continuously present in the indoor environment to which the subjects have been sensitized. In particular, the effects of co-exposure to both LRI and atopic stimuli in the same individual may be multiplicative, thus markedly intensifying the risk of development of persistent wheeze, which is consistent with the findings shown in table 4 . Such interactions are comparable to those recently described in studies performed on older schoolchildren, in whom viral infections have been shown to trigger asthma attacks, but with a high level of selectivity for subjects with pre-existing atopic sensitization to inhalants [26]. It should also be noted that these interactions do not occur between nonwheezing LRI and atopy, which is consistent with the belief that wheeze accompanying LRI represents 
Table 6. - Effect of exclusive breastfeeding for $<4$ months on current asthma after adjustment for various sets of confounders

Subjects $n$

\begin{tabular}{|c|c|c|c|}
\hline & \multirow[t]{2}{*}{ Subjects $n$} & \multicolumn{2}{|c|}{ Current asthma } \\
\hline & & OR $(95 \% \mathrm{CI})^{\#}$ & $\mathrm{p}$-value \\
\hline \multicolumn{4}{|l|}{ Model } \\
\hline a) Sex, gestational age, maternal smoking & 1871 & $1.19(0.93-1.53)$ & 0.170 \\
\hline b) Model a) and atopy & 1348 & $1.32(0.98-1.77)$ & 0.067 \\
\hline c) Model a) and infection exposure & 1836 & $1.18(0.92-1.52)$ & 0.194 \\
\hline d) Model b) and infection exposure & 1322 & $1.32(0.98-1.78)$ & 0.066 \\
\hline e) Model d) and maternal asthma & 1310 & $1.36(1.00-1.85)$ & 0.047 \\
\hline
\end{tabular}

a marker of potentially long-lasting damage to the growing airway, although it was not possible to examine this in the present study.

It has been suggested that wheezing LRI may serve either as a surrogate marker for small airway diameter or, alternatively, as an indicator of intense inflammatory damage to the airway, which has the potential to cause deleterious downstream effects on airway function at later ages $[6,25]$. Given that unrelated wheeze/ cough and doctor-diagnosis of asthma occurred 5-6 yrs after the wheezing LRI in question, at which time airway diameter would no longer be critically low, it appears more likely that the occurrence of wheezing LRI during infancy may be responsible for the long-term effects on airway function.

There also exists the intriguing possibility that individual causal pathways for the development of atopy and wheezing LRI may share common features and/or risk factors. In particular, it has previously been demonstrated that genetic risk for the development of atopy is associated with delayed postnatal development of the capacity to produce the T-helper (Th) 1 cytokine interferon (IFN)- $\gamma$ [24, 27], which antagonizes maturation of the Th2 cells responsible for atopic manifestations. This same cytokine plays a central role in defence against viral infections, including RSV, and recent studies indicate that wheezing bronchiolitis in infancy is associated with a reduced capacity to produce IFN- $\gamma[28,29]$.

The effects of respiratory infections on asthma development may, in some circumstances, be mediated via modulation of allergic sensitization to inhalants [24, 30-32]. However, in this large cohort, the effect of infections on current asthma appeared to be independent of skin-prick test reactivity, with the possible exception of two or more nonwheezing LRI. Nonwheezing LRIs were associated with reduced ORs for atopy (OR 0.62), although this did not reach statistical significance in this cohort. Here, it is relevant to mention the report of MARTINEZ et al. [33], in which nonwheezing LRI in the first 9 months of life were associated with reduced serum immunoglobulin-E levels at the age of 6 yrs.

Exclusive breastfeeding for $>4$ months was a significant protective factor for wheezing LRI, current asthma and atopy, following multivariate adjustment. The effect of exclusive breastfeeding was not considerably altered by adjustment of either atopy, infections (or both), or maternal asthma status, indicating that breastfeeding acts through other pathways to prevent asthma. The protective effects of a longer duration of breastfeeding are postulated to be mediated partially through the wheezing LRI pathway, for example, by provision of nutrients essential for the efficient operation of bioactivity and host-defence mechanisms [34] and, to a lesser degree, via the atopy pathway. However, in this study, the effects of breastfeeding on current asthma cannot entirely be accounted for by these mechanisms, indicating that it may influence other defence mechanisms, which are yet to be defined. One potential mechanism of the latter involves direct modulatory effects of breast milk on host inflammatory responses [35, 36], a possibility that merits further investigation. In addition, given the central importance of airway diameter in the overall wheezing process [37], future studies on the potential role of human milk in hastening the kinetics of lung growth during infancy appear to be warranted.

To conclude, this study provides new insight into the interactions between respiratory infections, atopy and breastfeeding in the aetiology of childhood asthma, and particularly illustrates potential pathway(s) via which the convergence of these risk factors may contribute to the development of persistent wheezing disease.

\section{References}

1. Hibbert M, Lannigan A, Raven J, Landau L, Phelan P. Gender differences in lung growth. Ped Pulmonol 1995; 19: 129-134.

2. Schwartz J, Weiss ST. Dietary factors and their relation to respiratory symptoms. The Second National Health and Nutrition Examination Survey. Am J Epidemiol 1990; 132: 67-76.

3. Rona RJ, Gulliford MC, Chinn S. Effects of prematurity and intrauterine growth on respiratory health and lung function in children. BMJ 1993; 306 : $817-820$.

4. Martinez FD, Wright AL, Holberg CJ, Morgan WJ, Taussig LM. Maternal age as a risk factor for wheezing lower respiratory illnesses in the first year of life. Am J Epidemiol 1992; 136: 1258-1268.

5. Wright AL, Holberg CJ, Taussig LM, Martinez FD. Factors influencing the relation of infant feeding to 
asthma and recurrent wheeze in childhood. Thorax 2001; 56: 192-197.

6. Martinez FD, Wright AL, Taussig LM, Holberg CJ, Halonen M, Morgan WJ. Asthma and wheezing in the first six years of life. $N$ Engl J Med 1995; 332: 133-138.

7. Peat JK, Tovey E, Toelle B, et al. House dust mite allergens: a major risk factor for childhood asthma in Australia. Am J Respir Crit Care Med 1996; 153: 141-146.

8. Holt PG. Environmental factors and primary T-cell sensitisation to inhalant allergens in infancy: reappraisal of the role of infections and air pollution. Ped Allergy Immunol 1995; 6: 1-10.

9. Wang SZ, Forsyth KD. Asthma and respiratory syncytial virus infection in infancy: is there a link? Clin Exp Allergy 1998; 28: 927-935.

10. Oddy WH, Holt PG, Sly PD, et al. Association between breastfeeding and asthma in 6 year old children: findings of a prospective birth cohort study. BMJ 1999; 319: 815-819.

11. Wilson AC, Stewart Forsyth J, Greene SA, Irvine L, Hau C, Howie PW. Relation of infant diet to childhood health: seven year follow up of cohort of children in Dundee infant feeding study. BMJ 1998; 316: 21-25.

12. Tariq SM, Matthews SM, Hakim EA, Stevens M, Arshad SH, Hide DW. The prevalence of and risk factors for atopy in early childhood: a whole population birth cohort study. J Allergy Clin Immunol 1998; 101: $587-593$.

13. Saarinen UM, Kajosaari M. Breastfeeding as prophylaxis against atopic disease: prospective follow-up study until 17 years old. Lancet 1995; 346: 1065-1069.

14. Johnston SL. Viruses and asthma. Allergy. Eur J Allergy Clin Immunol 1998; 53: 922-932.

15. Wright AL, Taussig LM, Ray GC, Harrison HR, Holberg CJ. The Tucson Children's Respiratory Study. II. Lower respiratory tract illness in the first year of life. Am J Epidemiol 1989; 129: 1232-1246.

16. Howie PW, Forsyth JS, Ogston SA, Clark A, Florey $\mathrm{CD}$. Protective effect of breastfeeding against infection. $B M J$ 1990; 300: 11-16.

17. Frank AL, Taber LH, Glezen WP, Kasel GL, Wells $\mathrm{CR}$, Paredes A. Breast feeding and respiratory virus infection. Pediatrics 1982; 70: 239-245.

18. Taylor B, Wadsworth J, Golding J, Butler N. Breast feeding, bronchitis, and admissions for lower respiratory illness and gastroenteritis during the first five years of life. Lancet 1982; 1: 1227-1229.

19. Newnham JP, Evans SF, Michael CA, Stanley FJ, Landau LI. Effects of frequent ultrasound during pregnancy: a randomised controlled trial. Lancet 1993; 342: 887-891

20. Dreborg S. Skin test in diagnosis of food allergy. Allergy Proc 1991; 12: 251-254.

21. Sigurs N, Bjarnason R, Sigurbergsson F, Kjellman B. Respiratory syncytial virus bronchiolitis in infancy is an important risk factor for asthma and allergy at age 7. Am J Respir Crit Care Med 2000; 161: 1501-1507.
22. von Mutius E, Illi S, Hirsch T, Leupold W, Keil U, Weiland SK. Frequency of infections and risk of asthma, atopy and airway hyperresponsiveness in children. Eur Respir J 1999; 14: 4-11.

23. Peat JK, Salome CM, Woolcock AJ. Longitudinal changes in atopy during a 4 year period: Relation to bronchial hyperresponsiveness and respiratory symptoms in a population sample of Australian school children. J Allergy Clin Immunol 1990; 85: 65-74.

24. Holt PG, Macaubas C, Stumbles PA, Sly PD. The role of allergy in the development of asthma. Nature 1999; 402: Suppl. 6760B, 12-17.

25. Welliver RC, Duffy L. The relationship of RSVspecific immunoglobulin $\mathrm{E}$ antibody responses in infancy, recurrent wheezing, and pulmonary function at age 7-8 years. Ped Pulmonol 1993; 15: 19-27.

26. Johnston SL, Pattemore PK, Sanderson G, et al. The relationship between upper respiratory infections and hospital admissions for asthma: A time-trend analysis. Am J Respir Crit Care Med 1996; 154: 654-660.

27. Holt PG, Clough JB, Holt BJ, et al. Genetic 'risk' for atopy is associated with delayed postnatal maturation of T-cell competence. Clin Exp Allergy 1992; 22: 1093-1099.

28. Aberle JH, Aberle SW, Dworzak MN, et al. Reduced interferon-gamma expression in peripheral blood mononuclear cells of infants with severe respiratory syncytial virus disease. Am J Respir Crit Care Med 1999; 160: 1263-1268.

29. Renzi PM, Turgeon JP, Marcotte JE, et al. Reduced interferon-gamma production in infants with bronchiolitis and asthma. Am J Respir Crit Care Med 1999; 159: $1417-1422$

30. Martinez FD. Role of viral infections in the inception of asthma and allergies during childhood: could they be protective? Thorax 1995; 49: 1189-1191.

31. Holt PG, Sly PD, Björksten B. Atopic versus infectious diseases in childhood: a question of balance? Ped Allergy Immunol 1997; 8: 53-58.

32. Martinez FD, Holt PG. The role of microbial burden in the aetiology of allergy and asthma. Lancet 1999; 354: Suppl. 11, 12-15.

33. Martinez FD, Stern DA, Wright AL, Taussig LM, Halonen M. Association of non-wheezing lower respiratory tract illnesses in early life with persistently diminished serum IgE levels. Group Health Medical Associates. Thorax 1995; 50: 1067-1072.

34. Hamosh M. Protective functions of proteins and lipids in human milk. Biol Neonate 1998; 74: 163-176.

35. Xanthou M. Immune protection of human milk. Biol Neonate 1998; 74: 121-133.

36. Hanson LÅ. Breastfeeding provides passive and likely longlasting active immunity. Ann Allergy Asthma Immunol 1998; 81: 523-537.

37. Martinez FD, Morgan WJ, Wright AL, Holberg CJ, Taussig LM. Diminished lung function as a predisposing factor for wheezing respiratory illness in infants. N Engl J Med 1988; 319: 1112-1117. 